This is an Open Access article distributed under the terms of the Creative Commons Attribution-Noncommercial License, which permits unrestricted use, distribution, and reproduction in any noncommercial medium, provided the original work is properly cited.

\title{
SCIENTIFIC GOALS OF SPICA FOR GALAXY FORMATION AND EVOLUTION IN EARLY UNIVERSE
}

\author{
Takashi Ichikawa, SPICA Task Force, and SPICA Science Team
}

\author{
Astronomical Institute, Tohoku University, Aoba, Sendai 980-8578, Japan
}

\begin{abstract}
We review the scientific goals of SPICA for galaxy formation and evolution in the early Universe, along with Japanese activities defining the goals and the specification of the relevant instruments. The main targets are: (1) discovery of first generation objects (Pop III stars) at the reionization epoch, (2) the origin of the cosmic farinfrared background and its fluctuation, (3) the star formation history of dusty galaxies and their contribution to stellar-mass assembly over the cosmic time, (4) the role of super-massive black holes in galaxy evolution, and (5) the effects of environment on galaxy evolution in cluster of galaxies and large scale structures at the high- $z$ Universe. The Japanese community for optical and infrared astronomy has organized a dedicated team for SPICA (SPICA Task Force, SFT). STF is playing a central role in defining the scientific capabilities of SPICA and in supporting the development of SPICA instruments: a large-format MIR camera, MIR spectrometers, and a high contrast photometric and spectroscopic MIR coronagraph.
\end{abstract}

Key words: Galaxies: formation - Galaxies: evolution Missions: SPICA

\section{SPICA TASK FORCE}

SPICA, which is nominated among the three most important astronomical future projects in Japan, is an earnest wish of Japanese astronomical community to be realized. GOPIRA (Group of Optical and Infrared Astronomy), which was originally organized by Japanese astronomers to construct the Subaru telescope, has considered SPICA as a next main goal along with the TMT after the success of Subaru project. To support SPICA project more strongly and intensively, GOPIRA has formed the SPICA Task Force (STF). On behalf of GOPIRA, the STF have discussed extensively the scientific goals, specifications of mid-infrared camera and spectrograph and coronagraph, collaborating with the SPICA science teams and the SPICA core group, In addition, one of its important roles has been to organize SPICA science workshops in Japan. All STF activities are devoted to the preparation for coming reviews of the SPICA project by JAXA, which are due in coming Fall (2009) and in 2010, to meet the full approval in 2011. In the preset paper, we briefly describe the scientific goals of SPICA for galaxy formation and evolution in early Universe, which have been discussed by the STF and SPICA science teams. We give a brief outline of the goals which are fully described in the SPICA Mission Requirement Document. The details are presented elsewhere in this volume (Egami; Shirahata; Nagao; Imanishi; Koyama).

\section{TOWARD the Epoch of Reionization}

We will identify and characterize dust-obscured IR luminous galaxies at $4<z<10$, where few far-IR/submm observations have hitherto explored. In so doing, we will target young galaxies, and then investigate how importantly IR galaxies played a role on re-ionization and galaxy formation in the early Universe.

Observational cosmology is now on the verge of making another great breakthrough in the history of science. The identification of the first generation of galaxies that appeared and reionized intergalactic space will end the so-called "Dark Age" of the Universe. The process, called "cosmic reionization", marks a major phase transition of the Universe, after which the intergalactic space has become transparent to $\mathrm{HI}$ ionizing radiation. Recent WMAP observations indicate that cosmic reionization took place around $z \sim 10$ (e.g., Dunkley et al. 2009) while QSO absorption-line studies suggest that the reionization process is not yet complete $z \sim 6$ (e.g., Fan et al. 2006). Our challenge, therefore, is to identify and characterize galaxies at $z>6$ that are responsible for this cosmic reionization.

Deep optical and near-/mid-infrared observations are now routinely detecting galaxies up to a redshift of $z \sim 7$, providing a first glimpse of galaxy populations near the epoch of reionization. Preliminary results suggest that the rest-frame UV luminosity density (and therefore the UVderived star formation rate density) is sharply declining from $z \sim 3-4$ to $z \sim 10$ (Bouwens et al. 2008). Such a decline in the rest-frame UV luminosity density was also seen when Lyman Break Galaxies (LBGs) were first studied at $z \sim 3-4$. The original decline is now understood as a result of inadequate dust-extinction correction. Furthermore, recent submillimetre observations found a population of heavily dust-obscured galaxies that are intrinsically luminous, but too faint in the optical to be detected in typ- 
ical $z \sim 3-4$ LBG surveys (e.g., Smail et al. 2002). This leads to a question that we hope to answer with SPICA: "Did dust-obscured (i.e., infrared-luminous) galaxies play an important role in the early history of the Universe at $4<z<10$ "

The IR/submm properties of galaxies at $4<z<10$ are virtually unknown. Therefore, SPICA's role will be twofold: (1) to identify $4<z<10$ IR-luminous galaxies, and (2) to characterize individual detected sources. For (2), we expect a number of interesting targets to be known beforehand from various JWST/ALMA deep surveys as well as from the SPICA surveys. We will also mention the great potential offered by lensing cluster surveys.

\section{Cosmic IR Background}

We will resolve cosmic far-infrared background light into individual objects, and reveal the origin of the residual background and its fluctuations.

Since the cosmic infrared-background radiation (CIB) was found with the COBE satellite (Puget et al. 1996; Hauser et al. 1998), it has been well- known that a large fraction of radiation energy in the Universe was released in far-infrared wavelengths. It has been suggested that one of the main sources of the far-infrared radiation is a result of the re-emission of UV light absorbed by dust in starforming galaxies at high redshift. Many previous efforts have been devoted to resolving the CIB into individual objects based on deep surveys with infrared space telescopes (e.g., ISO, Spitzer, and AKARI). While 10-30\% of the CIB has been resolved into individual galaxies, the origin of the rest is as yet unknown. Thanks to the highest sensitivity and highest spatial resolution of SPICA in mid-/far-infrared wavelengths, we expect to resolve CIB into individual sources hitherto unknown, which enable us to study the origin of CIB brightness and its fluctuations.

The scientific objectives of the study are (1) to determine the surface number density of infrared galaxies, (2) to determine the properties of individual galaxies identified, (3) to detect of faint-end flux in mid-/far-infrared wavelengths based on stacking analysis with deep images, and (4) to measure the absolute brightness and spatial fluctuations of CIB

SPICA is the telescope cooled to $6 \mathrm{~K}$ with aperture as large as $3.5 \mathrm{~m}$. As a result its sensitivity is two orders of magnitude higher than that of Herschel, and its spectral resolution is at least three times greater than that of AKARI and Spitzer. For point sources, SPICA is expected to resolve $90 \%$ and $60 \%$ of the CIB at $70 \mu \mathrm{m}$ and 160 $\mu \mathrm{m}$, respectively (Dole et al. 2004). From AKARI results, Shirahata et al. (2009) demonstrated the importance of multi-band number counts at $\sim 100 \mu \mathrm{m}$. Galaxy number counts from SPICA in the mid- to far-infrared will give us clues to understanding the evolutionary history of galaxies.
To know which galaxies dominate in their contribution to the CIB, the study of SEDs of detected sources will be very useful. An extra-galactic deep survey with AKARI discovered very young pure-starburst ULIRGs and extremely red objects (Takagi et al. 2009), which would be an evidence of the co-evolution of obscured AGN and starburst (Matsuhara et al. 2009). We will be able to detect statistically significant numbers of such galaxies beyond $z \sim 3$ by multi-band imaging observations with SAFARI and MIRACLE. A stacking analysis in comparison with the observations in shorter wavelengths would be effective in investigating the properties of unresolved sources, because confusion limits at the mid-infrared are much lower than that in the far-infrared.

\section{Metallicity of Young Galaxies}

We will study the metallicity of high-z dusty evolving galaxies, using MIR emission lines that originate from within dust-enshrouded galaxies.

The study of metallicity in galaxies at various redshifts will give a clue to evolutionary scenarios of galaxies as a function of redshift, because the chemical enrichment was expected from the consequence of their past star formation history. For example, a tight correlation of mass with metallicity (MZR) in galaxies (i.e., more massive galaxies have higher metallicity) suggests that the blow-off of gas with metals, which enriched in galaxies, by galactic superwinds is more efficient in less-massive galaxies (e.g., Tremonti et al. 2004). A more interesting issue would be the redshift evolution of MZR. Maiolino et al. (2008) reported a mass-dependent evolution of MZR at $0<z<3$; less-massive galaxies show gradual increase of metallicity from high to low redshift, while the enrichment of metals in massive galaxies ceased at earlier cosmic time. The phenomenon could be a sort of "down-sizing" in the chemical evolution of galaxies, analogous to down-sizing of stellar mass in galaxies. However, previous studies have been mainly based on UV-selected galaxies, which are not significantly obscured with dust.

In contrast, dust-rich ULIRGs are significantly deviated from MZR of UV selected galaxies (Rupke et al. 2008; Caputi et al. 2008). Since the ULIRGs are supposed to be in a rapidly evolving phase, MZR (and its redshift evolution) will give a clue to understanding galaxy evolution in the active star-forming epoch at high redshift, when active star formation occurred. It should be also noted that the previous studies of metallicity in ULIRGs have relied on rest-frame optical emission lines emitted in lessobscured regions. There have been few measurements in dust-enshrouded ULIRGs.

Observations with SPICA/SAFARI of the mid-/farinfrared metal lines emitted in dust-obscured region in ULIRGs at high redshift will solve the problem. Among the various diagnostics for metallicity, the emission-line flux ratios of [NIII] $(57.2 \mu \mathrm{m})$ to other lines are the most 
promising. For example, the flux ratio of [OIII] $(51.8 \mu \mathrm{m})$ / [NIII] $(57.2 \mu \mathrm{m})$ is very sensitive to gas metallicity. The complementary use of [OIII] $(88.3 \mu \mathrm{m})$, [OIII] $(51.8 \mu \mathrm{m})$, and [NIII] $(57.2 \mu \mathrm{m})$ is more effective, because the density dependence of the diagnostic ratio is well-corrected. The method is advantageous over other near- and mid-infrared metallicity diagnostics which make use of hydrogen lines (e.g., Verma et al. 2003), because infrared hydrogen lines such as Pfund and Humphreys series are generally very weak.

[OIII] to [NIII] line ratios from ISO data have been used to obtain the metallicity of Galactic planetary nebulae. The superb sensitivity of SPICA/SAFARI will enable us to extend such an approach to the extragalactic Universe. We have confirmed that the flux ratio of [OIII] 51.8 $\mu \mathrm{m}+88.3 \mu \mathrm{m}$ to [NIII] $57.2 \mu \mathrm{m}$ works very well as a metallicity diagnostic tool also for star-forming galaxies, based on detail photoionization model calculations (Nagao et al. in preparation). Note that these three lines are among strongest emission lines from HII regions in galaxies

Here we comment on the detection feasibility. The [NIII] $57.2 \mu \mathrm{m}$ line, which is faintest among the three lines, from galaxies resembling M82 in star-formation rate (Colbert et al. 1999) can be detected out to $z \sim 0.4$ with SPICA/SAFARI (5- $\sigma$ detection with one hour exposure). However, since ULIRGs, which are our main targets, have about SFRs two orders higher than M82, we will be able to observe [NIII] $57.2 \mu \mathrm{m}$ and two [OII] lines simultaneously from ULIRGs at $z \sim 2$. Note that a wavelength resolution of $\sim 1000$ is adequate for the present purpose, given a typical emission-line velocity width $\sim 300 \mathrm{~km} \mathrm{~s}^{-1}$ in starforming galaxies. In summary, SPICA/SAFARI will be capable of measuring the metallicity of ULIRGs at $z \sim 2$, using reddening-insensitive mid-infrared lines of [OIII] and [NIII]. The study is crucial to establishing the redshift evolution of MZR of rapidly evolving galaxies, and in this way SPICA will provide strong constraints on galaxy evolutionary scenarios.

\section{Mass Growth of Super Massive BH}

To explore the role of supper massive black holes (SMBHs) in galaxy evolution, we will conduct an extensive survey for actively mass-accreting SMBHs deeply buried in dust in the early Universe at $z>1$, when both star-formation and SMBH mass growth were at their peak.

Recent studies have shown that most if not all spheroid galaxies (bulges and elliptical galaxies) host a super massive black hole $(\mathrm{SMBH})$ in their centres and that the mass of the spheroid stars and SMBHs are tightly correlated. The findings strongly suggest that star-formation in the central few-kpc region of galaxies and SMBH mass growth, which occurred in the region smaller than 1 pc as AGN activity, are closely linked. Understanding the co-evolution of spheroid and SMBH over the cosmic history is one of the most fascinating topics in current astronomy. The cosmic energy radiation density, which is the sum of starformation and AGN activity, is higher in the infrared than in the UV/optical. Therefore, ongoing energy production is probably hidden behind dust. At $z>1$, ULIRGs with $\mathrm{L}(\mathrm{IR})>10^{\sim 12} L_{\odot}$ dominate cosmic infrared radiation. The probe of dust-obscured hidden energy sources in ULIRG population will lead to clarifying the history of coeval starformation activity and SMBH-mass growth and the conspiracy of galaxies and AGNs.

Unlike AGNs surrounded by torus-shaped dusty materials, which are classified optically as Seyferts, putative AGNs in ULIRG's nuclei are deeply buried in dust, hidden from any views, and therefore would be hardly observed in the optical. Infrared low-resolution $(R \sim 100)$ spectroscopy between $3-35 \mu \mathrm{m}$ is an effective means by which to investigate such optically-elusive AGNs in ULIRG nuclei, because the effect of dust extinction is negligibly small. In addition, we can distinguish star-formation phenomena from buried AGNs by infrared spectral shapes. Using the Subaru 8.2m telescope, Spitzer and AKARI infrared satellites, nearby many ULIRGs at $z<0.3$ have been investigated spectroscopically. We have found that (1) luminous buried AGNs are common in nearby ULIRGs, which are classified optically as non-Seyferts (HII-region or LINERs), and (2) buried AGNs are more energetic in galaxies with higher infrared luminosity and higher star formation rate (Imanishi et al. 2006; Imanishi et al. 2007; Imanishi et al. 2008; Imanishi 2009). The results are in good agreement with the well-known scenario that galaxydownsizing phenomenon is caused by stronger feedbacks from buried AGNs, which expel or heat surrounding gas in galaxies and stop further star formation in shorter time.

Both star-formation and SMBH-mass growth have peaks at $z>1$, so that it would be very important to directly investigate the conspiracy of galaxies and AGNs in this epoch, using an energy-diagnostic method along with restframe 3-35 $\mu \mathrm{m}$ spectroscopy. Infrared low-resolution $(R \sim$ 100) spectroscopy at $6-200 \mu \mathrm{m}$ in the observed frame, which is anticipated in SPICA with SAFARI and MIRACLE, will be the most promising technique for such studies. In fact, Herschel lacks the capability of wide-band and high-sensitivity spectroscopy. Studies of ULIRGs at shorter wavelengths with JWST will be severely hampered by the serious effects of dust extinction. Since ULIRGs are bright enough in the infrared, SPICA and low-resolution spectrographs will be advantageous for the study of star formation and AGNs in a statistically robust number of distant ULIRGs at $z>1$ within a reasonable amount of telescope time. Such observations would be difficult at other wavelengths like the (sub)millimetre (with ALMA).

\section{Cosmic Star-Formation and Mass Assembly}

We will study the star formation and mass assembly history of galaxies in relation to the formation of clusters 
of galaxies and large scale structures, as well as to the environmental effect on galaxy evolution.

The fifth goal is to understand the cosmic star formation history and mass assembly of the Universe. It is wellknown that cosmic star-formation rate density is higher at the higher redshift Universe with its peak at $z \sim 1-2$. However, surveys hitherto made at $z>1$ universe mainly rely on observations with rest-frame UV light, and these tend to be veiled behind dust. Therefore MIR-FIR observations have been waiting for the study of "hidden" starformation. Looking back to the distant Universe in the MIR-FIR is a fundamental approach to understand "real" star-formation history of the Universe. However, the situation is even more complicated because galaxy properties e.g. morphology, color, and star-formation rate, are strongly correlation with environment.

In the local Universe, galaxies in high-density environment such as in clusters have little star formation activity, though this is not necessarily true in the distant Universe. For example, a wide-field MIR study of a distant cluster of galaxies at $z \sim 0.8$ with AKARI has found a possible peak of star-forming activity at "intermediate-density" environment of cluster outskirts, groups and filaments (Koyama et al. 2008). Strong dusty starbursts are distributed along large-scale structures around clusters of galaxies. This fact suggests that the environment, where star formation is most active, changed with redshift and that the star formation is strongly correlated with the formation of largescale structures. We therefore need to explore star formation activity not only in clusters or in the general field, but also along large-scale structures at high redshift. Taking advantages of SIPCA, e.g., wide-field capability in MIR (MIRACLE) and excellent sensitivity at $>20 \mu \mathrm{m}$ (MIRACLE/SAFARI), we can study star-formation in dusty galaxies to $z \sim 5$ with rest-frame $7.7 \mu \mathrm{m}$ PAH emission, which is a good tracer of star-formation in dusty environments. It is notable that the $6^{\prime} \times 6^{\prime}$ FoV of MIRACLE cover well the large-scale structure surrounding clusters at $z \sim 1-5$.

We should also note that SPICA has unique advantages over other IR missions for studies across all redshifts. At $z<2$, the rest-frame $7.7 \mu \mathrm{m}$ PAH shifts to $\sim 10-25$ $\mu \mathrm{m}$, which will also be covered by the JWST. However, the small field coverage of JWST will not allow the tracing out of wide areas around large clusters. SPICA will be capable of observing the outskirts of cluster of galaxies and large scale structures, then construct a large sample of galaxies at various environments, which will give us clues to understanding the environment effect in a wide redshift range. We will also target "proto-clusters" at $z>2$, which are in early stage of clusters of galaxies in the present day, where many massive galaxies are expected to have been formed and extensive star formation in dusty galaxies took place.

We will be able to detect dusty galaxies in undergoing active star formation at $z \sim 3$ with MIRACLE in 20-40 $\mu \mathrm{m}$. In addition, the excellent sensitivity at $>40 \mu \mathrm{m}$ of
SAFARI will give us a great opportunity to extend our study up to $z \sim 5$. Mapping of very active star-forming ULIRGs in large scale structure at high- $z$ Universe is an exciting step for the study of star formation at high redshift. SPICA will be only instrument, which can devote to such studies. We will look back to the dusty Universe across wide range of environment based on the common tracers, such like $7.7 \mu \mathrm{m} \mathrm{PAH}$, of dusty star formation up to $z \sim 5$. We will unveil hidden activity of galaxies in conjunction with cluster formation history by exploring distant galaxy clusters and proto-clusters at $z \sim 1-5$ covering their outskirts.

SPICA will be able to efficiently study large samples of galaxies in different environments at various redshift. This will provide another parameter in the plot of cosmic star-formation history, which is an important step towards the complete understanding of the history of the evolution of galaxies.

\section{ACKNOWLEDGEMENTS}

This work has been supported in part by a Grant-in-Aid for Scientific Research (21244012) of the Ministry of Education, Culture, Sports, Science and Technology in Japan.

\section{REFERENCES}

Caputi, K. I., et al. 2008, ApJ, 680, 939

Colbert, E. J. M., et al. 1999, ApJ, 511, 721

Dole, H., et al. 2004, ApJS, 154, 93

Dunkley, J., et al. 2009, ApJS, 180, 306

Fan, X., et al. 2006, AJ, 132, 117

Hauser, M. G., et al. 1998, ApJ, 508, 25

Imanishi, M., et al. 2006, ApJ, 637, 114

Imanishi, M., et al. 2007, ApJS, 171, 72

Imanishi, M., et al. 2008, PASJ, 60, S489

Imanishi, M. 2006, ApJ, 694, 751

Koyama, Y., et al. 2009, MNRAS, 391, 1758

Matsuhara, H., et al. 2009, in preparation

Maiolino, R., et al. 2008, A\&Ap, 488, 463

Puget, J. -L., et al. 1996, A\&Ap, 308, 5

Rupke, D. S. N., et al. 2008, ApJ, 674, 51

Shirahata, M., et al. 2009, PASJ, 61, 737

Smail, I., et al. 2002, MNRAS, 331, 495

Takagi, T., et al. 2009, in preparation

Tremonti, C. A., et al. 2004, AJ, 613, 898

Verma, M. K., et al. 2003, A\&Ap, 403, 829 